\title{
A Risk-Based Interval Two-Stage Programming Model for Agricultural System Management under Uncertainty
}

\author{
Ye Xu${ }^{1}$ and Guohe Huang ${ }^{2}$ \\ ${ }^{1}$ MOE Key Laboratory of Regional Energy and Environmental Systems Optimization, \\ Resources and Environmental Research Academy, North China Electric Power University, Beijing 102206, China \\ ${ }^{2}$ Faculty of Engineering, University of Regina, Regina, SK, Canada S4S OA2 \\ Correspondence should be addressed to Ye Xu; xuye@ncepu.edu.cn
}

Received 17 March 2016; Revised 14 June 2016; Accepted 29 June 2016

Academic Editor: Vyacheslav Kalashnikov

Copyright ( $\odot 2016$ Y. Xu and G. Huang. This is an open access article distributed under the Creative Commons Attribution License, which permits unrestricted use, distribution, and reproduction in any medium, provided the original work is properly cited.

\begin{abstract}
Nonpoint source (NPS) pollution caused by agricultural activities is main reason that water quality in watershed becomes worse, even leading to deterioration. Moreover, pollution control is accompanied with revenue's fall for agricultural system. How to design and generate a cost-effective and environmentally friendly agricultural production pattern is a critical issue for local managers. In this study, a risk-based interval two-stage programming model (RBITSP) was developed. Compared to general ITSP model, significant contribution made by RBITSP model was that it emphasized importance of financial risk under various probabilistic levels, rather than only being concentrated on expected economic benefit, where risk is expressed as the probability of not meeting target profit under each individual scenario realization. This way effectively avoided solutions' inaccuracy caused by traditional expected objective function and generated a variety of solutions through adjusting weight coefficients, which reflected trade-off between system economy and reliability. A case study of agricultural production management with the Tai Lake watershed was used to demonstrate superiority of proposed model. Obtained results could be a base for designing land-structure adjustment patterns and farmland retirement schemes and realizing balance of system benefit, system-failure risk, and water-body protection.
\end{abstract}

\section{Introduction}

As a water-based compound system which is composed of natural, economical, social, and environmental factors, watershed has always been playing important roles in human survival and development. As rapid socioeconomical development, water shortage and water pollution issue related to watershed becomes more and more serious, which is an obstacle for coordinated development of social economy and ecological environment. To tackle such a problem, many types of point source control measures made large contribution for decreasing pollutants-discharging amounts, such as real-time monitoring, adoption of treatment, and control techniques, as well as design and implementation of some laws or regulations. However, water quality in watershed is not significantly improved, because NPS pollution issues have not been resolved well, especially pollution caused by agricultural activities [1].
NPS pollution issues sourced from agricultural production are presented as many aspects and ways, including soil erosion and loss, untreated rural domestic sewage and garbage, and unused nitrogen and phosphorus from fertilizer and manure. Moreover, the difficulties in controlling agricultural NPS pollution are exacerbated due to its some characteristics, such as diversities of discharge sources, disparity of spatial and temporal distribution, complexity of generation mechanism, uncertainty on load estimation, and unpopularity caused by overhigh control cost $[1,2]$. Therefore, how to generate an economy-effective and environmentally friendly agricultural production pattern under complexity and uncertainty is a critical issue for local authorities.

As demonstrated in previous studies [2-17], uncertain optimization techniques are suitable in tackling water quality management problems, which included stochastic mathematical programming (SMP), fuzzy mathematical programming (FMP), and interval linear programming (ILP), as well 
as their integrations. Among above optimization approaches, inexact two-stage stochastic programming (ITSP) model proposed by Huang and Loucks [18] was frequently applied in water management fields $[15,19-23]$. This is mainly due to the facts that (i) uncertain factors included within water quality management system exhibit random features, such as rainfall and runoff amounts; (ii) some factors are suitably assumed as interval formats due to their small variation range and limited information, including unit costs/benefits of crops and pollutants-generation amounts; (iii) policy makers involving large agricultural production projects should firstly know available tillable land area and then make appropriate decisions (i.e., plant area for various land-use types) after identifying precipitation-occurrence information and determining whether discharge standards are violated. ITSP model is useful in tackling probability distribution and interval information and designing corrective actions after a random event has taken place, leading to optimal decision schemes. Nevertheless, ITSP model also has a drawback in model configuration and may affect its stability and reliability. It is reflected that objective function of ITSP model is to maximize (or minimize) expected value under various probabilistic scenarios without consideration of possible risk while objective value under some specific scenarios may be worse. This is because that realization of expected objective value is incapable of ensuring that objective values under each scenario are optimal, leading to low system stability. Moreover, this drawback may be aggravated while uncertainties are exerting on management system in real world. Therefore, the improvement in objective function is necessary.

This study aims to develop a risk-based interval two-stage programming model for generating an optimal agricultural production pattern within the Tai Lake watershed, which has realized balance between system economy and system-failure risk. RBITSP model incorporated risk-estimation measure into objective function and ensures optimality of objective value under each scenario. A variety of solutions are obtained through adjusting weight coefficients in objective function, which are suitable in evaluating trade-off between system economy and reliability. The rest of this paper is organized as follows: introduction in studied region and its environmental issue are described in Section 2. Section 3 presents formulation of agricultural system optimization model and its solution algorithm. Result analysis, its comparison situation with traditional ITSP model, and potential improvement are demonstrated in Section 4. The summary will be provided in Section 5 .

\section{Case Study}

2.1. Introduction in the Tai Lake Watershed. Located in transit zone of northern subtropics and midsubtropics, Tai Lake watershed is a tributary in the lower reaches of Yangtze River, where Huangpu River is its main drainage channel (shown in Figure 1). The area of Tai Lake watershed is $36895 \mathrm{~km}^{2}$ approximately with numerous branching lakes. There are about 189 lakes distributing in Tai Lake watershed and their total area reached $3231 \mathrm{~km}^{2}$. The average temperature is $15-17^{\circ} \mathrm{C}$ and multiyear average precipitation is $1180 \mathrm{~mm}$

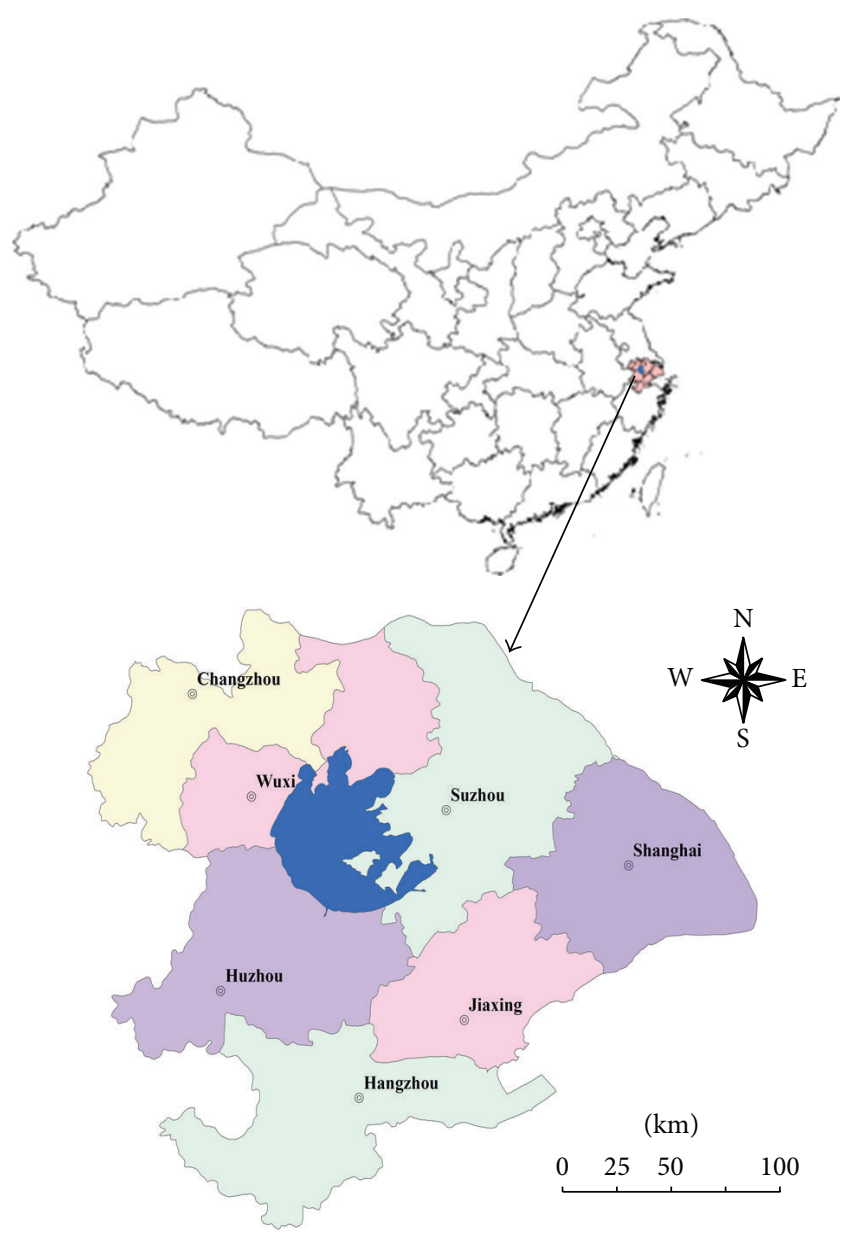

Figure 1: Studied region of Tai Lake watershed.

roughly. The landscape of this region is divided into two types: hills and plain, respectively. The plains are accounting for $80 \%$ of total area. The superior climate and geographical conditions of Tai Lake watershed, including sufficient light, heat, water, and land resources, are very beneficial for promoting development of agricultural economy. It makes watershed become core economic zones in China with the highest population concentration, economic scale, development potential, and innovation abilities and the Tai Lake watershed thus holds a critically important strategic position.

However, rapidly socioeconomical development not only realized the increase in economic output, but also has resulted in serious water pollutions. In recent years, frequent sudden water pollution accidents have brought serious damage to water environment and human's daily lives. Water pollution has become key factor limiting social and economic development of Tai Lake watershed. There are 35 observation sections selected for reflecting water quality situation in Tai Lake watershed [24]. As shown in monitoring results in May 2010, three sections meet requirement of the class II water quality standard; seven sections satisfied the class III water quality standard. Other 25 monitoring sections did not reach the class III water quality standard, of which four sections reached the class IV water quality standard, nine sections 
satisfied the class $\mathrm{V}$ water quality standard (which accounted for $25.7 \%$ ), and twelve sections were even worse than the class $\mathrm{V}$ water quality standard (which accounted for $34.3 \%$ ). The water quality evaluation is based on the "Environmental Quality Standards for Surface Water” (GB3838-2002) regulated by the national environmental protection agency, where surface water is classified into five types. Among them, the class I water means water quality is good enough to be drinking water; conversely, with gradual degradation in water quality, the class $\mathrm{V}$ water is provided for agricultural production due to its worse quality.

In recent few decades, control activities in point source pollutions effectively alleviate pollution level of point sources; water pollution caused by agricultural NPS pollutions become obvious, which have become main pollution source to Tai Lake watershed. The NPS pollutions of Tai Lake watershed are mainly reflected in following four aspects [25]: (i) overuse of fertilizer. As shown in Xia [24], average fertilizer-utilization amounts in Tai Lake watershed have increased from $224.5 \mathrm{~kg} / \mathrm{hm}^{2}$ in 1979 to $667.5 \mathrm{~kg} / \mathrm{hm}^{2}$ in 2014. Average proportion of fertilizer utilization is ranged in [0.3, 0.35]. The runoffs with large amount of fertilizer flow into water body, which results in water-quality degradation; (ii) soil erosion in western part bringing pollutants to water body. Currently, the area of soil erosion in Tai Lake watershed has reached $1472 \mathrm{~km}^{2}$; (iii) pollution caused by livestock breeding. With improvement of living quality and production structure adjustment, livestock-breeding scale is increasing continually. Recently, total number of livestock and poultry has reached 347 million; (iv) other pollution sources, including agricultural residues, rural domestic wastes, biological pollutions deriving from excessive growth, and rot of aquatic plants, as well as poor management of infrastructure constructions and operations. Therefore, effective control and management of NPS pollution have become the key for solving water pollution issue in Tai Lake watershed.

Previous studies and practices indicate that most economical and effective way to control NPS pollutions is realizing source control, which mainly includes scientific use of fertilizers, adjusting land-use types, as well as enhancing waste management of livestock breeding. Among them, the change in land-use types has very important influences on reduction of NPS pollutions $[22,26]$. Through adjusting plant structure, cultivating area of crops with the high pollutants emissions can be limited and the amounts of soil erosions and pollutants flowing into water body can be reduced, which is useful in controlling water pollution. On the other hand, the adjustment of agricultural structures is difficult to be realized due to its negative effects on agricultural production benefits. Therefore, how to maximize economical performance of agricultural structures adjustment and meanwhile make pollutant emissions reach designed standard have become a critical issue for watershed development. Optimization model is capable of tackling this problem.

2.2. Understanding and Identification of System Configuration and Parameters. In this study, agricultural system management issue of Tai Lake watershed is selected as studied target in order to provide decision support for coordinated development of watershed. Generally, watershed is composed of multiple subsystems, such as natural, social, economic, ecoenvironmental, and water resource subsystems. All subsystems are interrelated and interacted with each other. For example, the determination of crop area is affected by many factors, including available land area, economical output, regulated pollutants-discharge amounts, and water provision amounts. Once cropping areas are fixed, they in turn affected reserves of land and water resources, water quality, and ecological balance, as well as living quality of local residents. Similarly, strict pollutants-discharge standards are necessary in order to realize environmental quality improvement. However, they also may lead to decrease in revenues due to their limitations in agricultural activities and will be met with opposition by local residents. From the point of systemic view, deep understanding in system framework and operation is critical for generating ideal agricultural production patterns.

In decision-making process, five agricultural zones in Tai Lake watershed were selected, adjustment of agricultural production structures is examined, and discharge control of total nitrogen and total phosphorus (i.e., TN and TP) was considered. According to variations in precipitation amounts, planning horizon is designed as three periods where each period is four months, that is, dry, medium, and wet seasons. As demonstrated in Section 1, there are many uncertain factors existing in management system. Accurate identification and expression in such uncertainties are very important in order to establish a rational agricultural production management model. As shown in statistical results of historical observation data, precipitation amounts follow probabilistic distributions and are thus assumed as fixed values under a specific scenario set. Table 1 provides the details. Other factors, which are related to environmental parameters, including pollutants-generation amounts and allowable discharge amounts, are varying in small range and are thus assumed as interval numbers. Their introductions and descriptions are reflected in Table 2. With regard to some economical parameters, such as agricultural production revenues and treated costs for excess discharge of pollutants, they also are expressed as discrete intervals and are depicted in Table 3. The related data information is referring to Liu et al. [22].

\section{Methodology}

3.1. Model Formulation for Agricultural Production Management. Within the context of agricultural management, agricultural production department should know in advance available land areas for various land-use types, that is, how much area of land could be used to be crop and forestland, respectively. According to the information provided by local authorities, production sectors will design appropriate investment plans in irrigation infrastructure for realizing maximum production profits. Moreover, based on predefined land-allocation target, local manager should ensure that available land be effectively utilized with the minimization of any associated penalties, where penalties mean overhigh 
TABLE 1: Precipitation amounts under various probabilistic levels.

\begin{tabular}{|c|c|c|c|c|c|c|}
\hline \multirow[b]{2}{*}{ Zone $(i)$} & \multicolumn{6}{|c|}{ Precipitation amounts at three periods (mm) } \\
\hline & $\begin{array}{c}\text { Probability } \\
\text { level }(j)\end{array}$ & $\begin{array}{c}\text { Planning } \\
\text { period }(k=1)\end{array}$ & $\begin{array}{c}\text { Probability } \\
\text { level }(j)\end{array}$ & $\begin{array}{c}\text { Planning } \\
\text { period }(k=2)\end{array}$ & $\begin{array}{l}\text { Probability } \\
\text { level }(j)\end{array}$ & $\begin{array}{c}\text { Planning } \\
\text { period }(k=3)\end{array}$ \\
\hline \multirow{3}{*}{$i=1$} & $P=0.15$ & {$[130,150]$} & $P=0.17$ & {$[280,315]$} & $P=0.15$ & {$[680,730]$} \\
\hline & $P=0.70$ & {$[160,180]$} & $P=0.66$ & {$[330,365]$} & $P=0.70$ & {$[750,800]$} \\
\hline & $P=0.15$ & {$[190,210]$} & $P=0.17$ & {$[380,415]$} & $P=0.15$ & {$[820,870]$} \\
\hline \multirow{3}{*}{$i=2$} & $P=0.20$ & {$[145,165]$} & $P=0.12$ & {$[310,345]$} & $P=0.14$ & {$[740,790]$} \\
\hline & $P=0.60$ & {$[175,195]$} & $P=0.76$ & {$[360,395]$} & $P=0.72$ & {$[810,860]$} \\
\hline & $P=0.20$ & {$[205,225]$} & $P=0.12$ & {$[410,445]$} & $P=0.14$ & {$[880,930]$} \\
\hline \multirow{3}{*}{$i=3$} & $P=0.10$ & {$[165,185]$} & $P=0.11$ & {$[350,385]$} & $P=0.15$ & {$[830,880]$} \\
\hline & $P=0.80$ & {$[195,215]$} & $P=0.78$ & {$[400,435]$} & $P=0.70$ & {$[900,950]$} \\
\hline & $P=0.10$ & {$[225,245]$} & $P=0.11$ & {$[450,485]$} & $P=0.15$ & {$[970,1020]$} \\
\hline \multirow{3}{*}{$i=4$} & $P=0.14$ & {$[110,130]$} & $P=0.22$ & {$[250,285]$} & $P=0.14$ & {$[600,650]$} \\
\hline & $P=0.72$ & {$[140,160]$} & $P=0.56$ & {$[300,335]$} & $P=0.72$ & {$[670,720]$} \\
\hline & $P=0.14$ & {$[170,190]$} & $P=0.22$ & {$[350,385]$} & $P=0.14$ & {$[740,790]$} \\
\hline \multirow{3}{*}{$i=5$} & $P=0.18$ & {$[95,115]$} & $P=0.15$ & {$[220,255]$} & $P=0.13$ & {$[540,590]$} \\
\hline & $P=0.64$ & {$[125,145]$} & $P=0.70$ & {$[270,305]$} & $P=0.74$ & {$[610,660]$} \\
\hline & $P=0.18$ & {$[155,175]$} & $P=0.15$ & {$[320,355]$} & $P=0.13$ & {$[680,730]$} \\
\hline
\end{tabular}

Note: the related data are referred to Liu et al. [22].

TABLE 2: Environmental parameters presented as discrete intervals.

\begin{tabular}{|c|c|c|c|c|c|}
\hline \multirow{2}{*}{ Planning period $(k)$} & \multicolumn{5}{|c|}{ Agricultural zone $(i)$} \\
\hline & $i=1$ & $i=2$ & $i=3$ & $i=4$ & $i=5$ \\
\hline \multicolumn{6}{|c|}{ The concentrations of the pollutant TN in the surface runoff from cropland (mg/L) } \\
\hline$k=1$ & {$[1.25,1.45]$} & {$[0.95,1.15]$} & {$[1.35,1.55]$} & {$[0.90,1.10]$} & {$[1.10,1.30]$} \\
\hline$k=2$ & {$[2.15,2.50]$} & {$[1.80,2.15]$} & {$[2.50,2.85]$} & {$[1.65,2.00]$} & {$[2.05,2.40]$} \\
\hline$k=3$ & {$[4.20,4.65]$} & {$[3.45,3.90]$} & {$[4.55,5.00]$} & {$[3.10,3.55]$} & {$[3.95,4.40]$} \\
\hline \multicolumn{6}{|c|}{ The concentrations of the pollutant TP in the surface runoff from cropland (mg/L) } \\
\hline$k=1$ & {$[0.14,0.16]$} & {$[0.11,0.13]$} & {$[0.16,0.18]$} & {$[0.09,0.11]$} & {$[0.13,0.15]$} \\
\hline$k=2$ & {$[0.24,0.27]$} & {$[0.20,0.23]$} & {$[0.26,0.29]$} & {$[0.17,0.20]$} & {$[0.23,0.26]$} \\
\hline$k=3$ & {$[0.44,0.49]$} & {$[0.40,0.45]$} & {$[0.46,0.51]$} & {$[0.34,0.39]$} & {$[0.43,0.48]$} \\
\hline \multicolumn{6}{|c|}{ The concentrations of the pollutant TN in the surface runoff from forestland $(\mathrm{mg} / \mathrm{L})$} \\
\hline$k=1$ & {$[0.55,0.65]$} & {$[0.60,0.70]$} & {$[0.75,0.85]$} & {$[0.50,0.60]$} & {$[0.50,0.60]$} \\
\hline$k=2$ & {$[0.95,1.15]$} & {$[1.10,1.30]$} & {$[1.20,1.40]$} & {$[0.85,1.05]$} & {$[0.80,1.00]$} \\
\hline$k=3$ & {$[1.65,1.95]$} & {$[1.90,2.20]$} & {$[2.15,2.45]$} & {$[1.50,1.80]$} & {$[1.40,1.70]$} \\
\hline \multicolumn{6}{|c|}{ The concentrations of the pollutant TP in the surface runoff from forestland $(\mathrm{mg} / \mathrm{L})$} \\
\hline$k=1$ & {$[0.03,0.04]$} & {$[0.04,0.05]$} & {$[0.04,0.05]$} & {$[0.03,0.04]$} & {$[0.03,0.04]$} \\
\hline$k=2$ & {$[0.06,0.08]$} & {$[0.07,0.09]$} & {$[0.07,0.09]$} & {$[0.05,0.07]$} & {$[0.04,0.06]$} \\
\hline$k=3$ & {$[0.09,0.12]$} & {$[0.11,0.14]$} & {$[0.12,0.15]$} & {$[0.09,0.12]$} & {$[0.08,0.11]$} \\
\hline
\end{tabular}

Note: the related data are referred to Liu et al. [22].

treatment costs for excess discharge of pollutants caused by agricultural production activities in order to maintain water quality of Tai Lake watershed. To tackle above problems, ITSP model is useful.

Under the context of ITSP framework of agricultural system management in Tai Lake watershed, the meaning of "two-stage" is that a decision of agricultural production pattern needs to be made at the first stage before precipitation amounts are known; a recourse action (i.e., adjustment of land-cultivation structure) can then be taken at the second stage, when uncertainties of pollutants-generation amounts are quantified and land-allocation targets are fixed. Objective function of ITSP model is determined as maximization of net system benefit, which is described as the difference between agricultural production revenues and cost caused by excess discharge of pollutants generated by agricultural 
TABLE 3: Economical parameters presented as discrete intervals.

\begin{tabular}{|c|c|c|c|c|c|}
\hline \multirow{2}{*}{ Economical parameters } & \multicolumn{5}{|c|}{ Agricultural zone (i) } \\
\hline & $i=1$ & $i=2$ & $i=3$ & $i=4$ & $i=5$ \\
\hline \multicolumn{6}{|c|}{ Unit net revenue of the agricultural production $(\$ /$ ha $)$} \\
\hline Cropland & {$[1820,1940]$} & {$[1545,1665]$} & {$[1700,1820]$} & {$[1630,1750]$} & {$[1440,1550]$} \\
\hline Forestland & {$[1050,1170]$} & {$[1220,1340]$} & {$[1270,1390]$} & {$[1060,1180]$} & {$[930,1040]$} \\
\hline \multicolumn{6}{|c|}{ The penalty costs for the excess discharge of the pollutant TN $(\$ / \mathrm{kg})$} \\
\hline$k=1$ & {$[19,25]$} & {$[21,28.5]$} & {$[20,26.5]$} & {$[18,23.5]$} & {$[18,23.5]$} \\
\hline$k=2$ & {$[21.5,28.5]$} & {$[24,32.5]$} & {$[22.5,30.5]$} & {$[20.5,27.5]$} & {$[21,27.5]$} \\
\hline$k=3$ & {$[23.5,33]$} & {$[26.5,36.5]$} & {$[25,34.5]$} & {$[23.5,31.5]$} & {$[24.5,31.5]$} \\
\hline \multicolumn{6}{|c|}{ The penalty costs for the excess discharge of the pollutant TP $(\$ / \mathrm{kg})$} \\
\hline$k=1$ & {$[39,51]$} & {$[43,58]$} & {$[41.5,53.5]$} & {$[38,49]$} & {$[38,49]$} \\
\hline$k=2$ & {$[44,56]$} & {$[49.5,63.5]$} & {$[47,58.5]$} & {$[43.5,54]$} & {$[43,54]$} \\
\hline$k=3$ & {$[51,60.5]$} & {$[56,68.5]$} & {$[53.5,63.5]$} & {$[50,59]$} & {$[48,59]$} \\
\hline
\end{tabular}

Note: the related data are referred to Liu et al. [22].

activities. Constraints mainly included available land-area restriction, limitation of pollutants-discharge amounts, and nonnegativity restrictions. An ITSP model for agricultural system management can be formulated as follows:

$$
\text { Maximize } f^{ \pm}=\sum_{i=1}^{I}\left(L B_{i}^{ \pm} L X_{i}^{ \pm}+F B_{i}^{ \pm} F Y_{i}^{ \pm}\right)-\sum_{i=1}^{I} \sum_{j=1}^{J} \sum_{k=1}^{K} P_{i j k}\left(C N_{i k}^{ \pm} Z_{n, i j k}^{ \pm}+C P_{i k}^{ \pm} Z_{p, i j k}^{ \pm}\right)
$$

where $f^{ \pm}$is expected agricultural production profit and expressed as interval form (\$), where items $f^{-}$and $f^{+}$in equation $f^{ \pm}=\left[f^{-}, f^{+}\right]$are the lower and upper bounds of $f^{ \pm}$, respectively; $L B_{i}^{ \pm}$and $F B_{i}^{ \pm}$are profits of cropland cultivation and forestland generated from zone $i(\$ /$ ha), respectively, where $i$ is name of zone $(i=1,2, \ldots, I) ; L X_{i}^{ \pm}$and $F Y_{i}^{ \pm}$are designed allocation targets of cropland and forestland at the first stage (ha), respectively; $P_{i j k}$ is probability of occurrence of specified precipitation level, where $j$ is precipitation level $(j=1,2, \ldots, J)$ and $k$ is time period $(k=1,2, \ldots, K) ; C N_{i k}^{ \pm}$ and $C P_{i k}^{ \pm}$are penalty costs for excess discharge of TN and TP generated from zone $i$ during period $k(\$ / \mathrm{kg})$, respectively; $Z_{n, i j k}^{ \pm}$and $Z_{p, i j k}^{ \pm}$are excess discharge amounts of TN and $\mathrm{TP}$ in zone $i$ during period $k$ under precipitation level $j$ $(\mathrm{kg})$, respectively. Objective function (1) is net benefits which equals agricultural production revenues minus pollutantstreatment costs.

Subject to the following:

(1) Land area balance

$$
L X_{i}^{ \pm}+F Y_{i}^{ \pm}=A_{i}, \quad \forall i
$$

where $A_{i}$ is total available land area (ha). Constraint (2) regulated that tillable land of studied agricultural region is occupied by cropland and forestland.

(2) Total nitrogen losses constraint

$$
\begin{aligned}
E_{n, i j k}^{ \pm}= & \mu_{i k}^{ \pm} \times L X_{i}^{ \pm} \times R_{i j k}^{ \pm} \times C_{n, i k}^{ \pm}+v_{i k}^{ \pm} \times F Y_{i}^{ \pm} \\
& \times R_{i j k}^{ \pm} \times D_{n, i k}^{ \pm}, \quad \forall i, j, k
\end{aligned}
$$

$$
\begin{aligned}
& 0 \leq Z_{n, i j k}^{ \pm} \leq E_{n, i j k}^{ \pm}, \quad \forall i, j, k \\
& E_{n, i j k}^{ \pm}-Z_{n, i j k}^{ \pm} \leq S_{n, i k}^{ \pm}, \quad \forall i, j, k \\
& \sum_{i=1}^{I} S_{n, i k}^{ \pm} \leq T_{n, k}^{ \pm}, \quad \forall k,
\end{aligned}
$$

where $E_{n, i j k}^{ \pm}$is discharge amounts of TN in zone $i$ during period $k$ under precipitation level $j(\mathrm{~kg}) ; \mu_{i k}^{ \pm}$and $v_{i k}^{ \pm}$are surface runoff coefficients in cropland and forestland, respectively; $R_{i j k}^{ \pm}$is precipitation amounts with probabilities $P_{i j k}$, $(\mathrm{mm}) ; C_{n, i k}^{ \pm}$and $D_{n, i k}^{ \pm}$are concentrations of TN in surface runoff from cropland and forestland $(\mathrm{mg} / \mathrm{L})$, respectively; $S_{n, i k}^{ \pm}$is reallocated discharge permits of TN in zone $i$ during period $k$ under the context of discharge trading $(\mathrm{kg}) ; T_{n, k}^{ \pm}$ is total discharge permits of TN for during period $k(\mathrm{~kg})$. Constraint (3) is used to calculate generation amounts of TN sourced from production activities, where surface runoff coefficients were designed as one percent of original parameters values in order to realize consistency of left and right sides units of constraint (3). Constraint (4) regulated relationships between generation and excess amounts. Constraints (5) and (6) required discharge amounts of pollutants $\mathrm{TN}$ are lower than allowable discharge amounts.

(3) Total phosphorus losses constraint

$$
\begin{aligned}
E_{p, i j k}^{ \pm}= & \mu_{i k}^{ \pm} \times L X_{i}^{ \pm} \times R_{i j k}^{ \pm} \times C_{p, i k}^{ \pm}+v_{i k}^{ \pm} \times F Y_{i}^{ \pm} \\
& \times R_{i j k}^{ \pm} \times D_{p, i k}^{ \pm}, \quad \forall i, j, k
\end{aligned}
$$




$$
\begin{aligned}
0 & \leq Z_{p, i j k}^{ \pm} \leq E_{p, i j k}^{ \pm}, \quad \forall i, j, k \\
E_{p, i j k}^{ \pm}-Z_{p, i j k}^{ \pm} & \leq S_{p, i k}^{ \pm}, \quad \forall i, j, k \\
\sum_{i=1}^{I} S_{p, i k}^{ \pm} & \leq T_{p, k}^{ \pm}, \quad \forall k
\end{aligned}
$$

where $E_{p, i j k}^{ \pm}$is discharge amounts of TP in zone $i$ during period $k$ under precipitation level $j(\mathrm{~kg}) ; C_{p, i k}^{ \pm}$and $D_{p, i k}^{ \pm}$are concentrations of TP in surface runoff from cropland and forestland $(\mathrm{mg} / \mathrm{L})$, respectively; $S_{p, i k}^{ \pm}$is reallocated discharge permits of TP in zone $i$ during period $k$ under the context of discharge trading $(\mathrm{kg}) ; T_{p, k}^{ \pm}$is total discharge permits of TN for during period $k(\mathrm{~kg})$. The meanings of constraints (7) are similar to some above constraints (3) to (6), correspondingly.

(4) Nonnegativity constraint

$$
L X_{i}^{ \pm}, F Y_{i}^{ \pm} \geq 0, \quad \forall i .
$$

As described in Section 1, the maximization of expected objective value is incapable of ensuring maximization of objective function values under each scenario. Therefore, financial risk associated with agricultural production should be incorporated into ITSP framework, which is capable of guaranteeing that objective value under each scenario reaches specific target as possible. Referring to Barbaro and Bagajewicz [27], financial risk can be defined as probability of not meeting a certain target profit level referred to as $\Omega_{q}$, where $q$ reflects various target values. For models (1)-(8), financial risk associated with decision variables and target profit $\Omega_{q}$ is expressed by probability measure [27]:

$$
\begin{aligned}
& \operatorname{Risk}\left(L X_{i}^{ \pm}, F Y_{i}^{ \pm}, Z_{n, i j k}^{ \pm}, Z_{p, i j k}^{ \pm}, \Omega_{q}\right) \\
& =P\left[\operatorname{Profit}\left(L X_{i}^{ \pm}, F Y_{i}^{ \pm}, Z_{n, i j k}^{ \pm}, Z_{p, i j k}^{ \pm}\right)<\Omega_{q}\right] \\
& =\sum_{q=1}^{Q} P\left(f^{ \pm}<\Omega_{q}\right) \\
& =\sum_{i=1}^{I} \sum_{j=1}^{J} \sum_{k=1}^{K} \sum_{q=1}^{Q} P_{i j k} w_{i j k, q}\left(L X_{i}^{ \pm}, F Y_{i}^{ \pm}, Z_{n, i j k}^{ \pm}, Z_{p, i j k}^{ \pm}, \Omega_{q}\right) \\
& w_{i j k, q}\left(L X_{i}^{ \pm}, F Y_{i}^{ \pm}, Z_{n, i j k}^{ \pm}, Z_{p, i j k}^{ \pm}, \Omega_{q}\right) \\
& =\left\{\begin{array}{ll}
1 & \text { if } f^{ \pm}<\Omega_{q} \\
0 & \text { otherwise }
\end{array} \forall i, j, k, q,\right.
\end{aligned}
$$

where $\Omega_{q}$ is predefined target profit and $q(q=1,2, \ldots, Q)$ is profit level and $w_{i j k, q}$ is binary variable, as shown in (9), where $w_{i j k, q}$ is zero when profit for zone $i$, scenario $j$, and period $k$ is greater than or equal to target level $\Omega_{q}$ and a value of one otherwise. In order to examine influences caused by possible financial risk, above two equations are incorporated into models (1)-(8), leading to a RBISTP model as follows:

$$
\begin{aligned}
& \text { Maximize } \quad f^{ \pm}=\sum_{i=1}^{I}\left(L B_{i}^{ \pm} L X_{i}^{ \pm}+F B_{i}^{ \pm} F Y_{i}^{ \pm}\right)-\sum_{i=1}^{I} \sum_{j=1}^{J} \sum_{k=1}^{K} P_{i j k}\left(C N_{i k}^{ \pm} Z_{n, i j k}^{ \pm}+C P_{i k}^{ \pm} Z_{p, j k}^{ \pm}\right)-\alpha_{m} \sum_{i=1}^{I} \sum_{j=1}^{J} \sum_{k=1}^{K} \sum_{q=1}^{Q} P_{i j k} w_{i j k, q} \\
& \text { Subject to: } L X_{i}^{ \pm}+F Y_{i}^{ \pm}=A_{i}, \quad \forall i \\
& E_{n, i j k}^{ \pm}=\mu_{i k}^{ \pm} \times L X_{i}^{ \pm} \times R_{i j k}^{ \pm} \times C_{n, i k}^{ \pm}+v_{i k}^{ \pm} \times F Y_{i}^{ \pm} \times R_{i j k}^{ \pm} \times D_{n, i k}^{ \pm}, \quad \forall i, j, k \\
& 0 \leq Z_{n, i j k}^{ \pm} \leq E_{n, i j k}^{ \pm}, \quad \forall i, j, k \\
& E_{n, i j k}^{ \pm}-Z_{n, i j k}^{ \pm} \leq S_{n, i k}^{ \pm}, \quad \forall i, j, k \\
& \sum_{i=1}^{I} S_{n, i k}^{ \pm} \leq T_{n, k}^{ \pm}, \quad \forall k \\
& E_{p, i j k}^{ \pm}=\mu_{i k}^{ \pm} \times L X_{i}^{ \pm} \times R_{i j k}^{ \pm} \times C_{p, i k}^{ \pm}+v_{i k}^{ \pm} \times F Y_{i}^{ \pm} \times R_{i j k}^{ \pm} \times D_{p, i k}^{ \pm}, \quad \forall i, j, k \\
& 0 \leq Z_{p, i j k}^{ \pm} \leq E_{p, i j k}^{ \pm}, \quad \forall i, j, k \\
& E_{p, i j k}^{ \pm}-Z_{p, i j k}^{ \pm} \leq S_{p, i k}^{ \pm}, \quad \forall i, j, k \\
& \sum_{i=1}^{I} S_{p, i k}^{ \pm} \leq T_{p, k}^{ \pm}, \quad \forall k \\
& w_{i j k, q}\left(L X_{i}^{ \pm}, F Y_{i}^{ \pm}, Z_{n, i j k}^{ \pm}, Z_{p, i j k}^{ \pm}, \Omega_{q}\right)=\left\{\begin{array}{ll}
1 & \text { if } f^{ \pm}<\Omega_{q} \\
0 & \text { otherwise }
\end{array} \quad \forall i, j, k, q\right. \\
& w_{i j k, q} \in(0,1) \quad \forall i, j, k, q \\
& L X_{i}^{ \pm}, F Y_{i}^{ \pm} \geq 0, \quad \forall i,
\end{aligned}
$$


where $\alpha_{m}$ is weight coefficient and $m$ represents attitude of decision makers on financial risk. $\Omega_{q}^{ \pm}$is assumed as interval values.

3.2. Solution Algorithm of Formulated Agricultural Management Model. To solve models (10)-(22), constraint (20) should be transformed into deterministic form firstly. Referring to Barbaro and Bagajewicz [27], constraint (20) can be rewritten as follows:

$$
\begin{aligned}
& \sum_{i=1}^{I}\left(L B_{i}^{ \pm} L X_{i}^{ \pm}+F B_{i}^{ \pm} F Y_{i}^{ \pm}\right) \\
& \quad-\sum_{i=1}^{I} \sum_{j=1}^{J} \sum_{k=1}^{K} P_{i j k}\left(C N_{i k}^{ \pm} Z_{n, i j k}^{ \pm}+C P_{i k}^{ \pm} Z_{p, i j k}^{ \pm}\right) \geq \Omega_{q}^{ \pm} \\
& \quad-U_{q} w_{i j k, q} \quad \forall i, j, k, q
\end{aligned}
$$

$$
\begin{aligned}
& \sum_{i=1}^{I}\left(L B_{i}^{ \pm} L X_{i}^{ \pm}+F B_{i}^{ \pm} F Y_{i}^{ \pm}\right) \\
& \quad-\sum_{i=1}^{I} \sum_{j=1}^{J} \sum_{k=1}^{K} P_{i j k}\left(C N_{i k}^{ \pm} Z_{n, i j k}^{ \pm}+C P_{i k}^{ \pm} Z_{p, i j k}^{ \pm}\right)<\Omega_{q}^{ \pm} \\
& \quad+U_{q}\left(1-w_{i j k, q}\right) \quad \forall i, j, k, q,
\end{aligned}
$$

where $U_{q}$ is predefined upper bound of profit under each scenario. Constraints (23) and (24) are used to ensure binary variable $w_{i j k, q}$ is zero or one when profit under scenario $q$ is greater than, equal to, or lower than target level $\Omega_{q}^{ \pm}$, respectively. After above transformation, models (10)-(22) is converted into ITSP model. Based on Huang and Loucks [18], let $L X_{i}^{ \pm}=L X_{i}^{-}+\Delta L X_{i} G_{i}$, where $\Delta L X_{i}=L X_{i}^{+}-L X_{i}^{-}$and $G_{i} \in[0,1] ; F Y_{i}^{ \pm}=F Y_{i}^{-}+\Delta F Y_{i} H_{i}$, where $\Delta F Y_{i}=F Y_{i}^{+}-F Y_{i}^{-}$ and $H_{i} \in[0,1]$. The variables $G_{i}$ and $H_{i}$ are decision variables, which are used to determine optimal sets of target values $L X_{i}$ and $F Y_{i}$, respectively. Finally, models (10)-(22) can be rewritten as follows:

$$
\begin{aligned}
& \text { Maximize } f^{ \pm} \\
& =\sum_{i=1}^{I}\left(L B_{i}^{ \pm}\left(L X_{i}^{-}+\Delta L X_{i} G_{i}\right)+F B_{i}^{ \pm}\left(F Y_{i}^{-}+\Delta F Y_{i} H_{i}\right)\right)-\sum_{i=1}^{I} \sum_{j=1}^{J} \sum_{k=1}^{K} P_{i j k}\left(C N_{i k}^{ \pm} Z_{n, i j k}^{ \pm}+C P_{i k}^{ \pm} Z_{p, i j k}^{ \pm}\right) \\
& -\alpha_{m} \sum_{i=1}^{I} \sum_{j=1}^{J} \sum_{k=1}^{K} \sum_{q=1}^{Q} P_{i j k} w_{i j k, q} \\
& \text { Subject to: } L X_{i}^{-}+\Delta L X_{i} G_{i}+F Y_{i}^{-}+\Delta F Y_{i} H_{i}=A_{i}, \quad \forall i \\
& E_{n, i j k}^{ \pm}=\mu_{i k}^{ \pm} \times L X_{i}^{ \pm} \times R_{i j k}^{ \pm} \times C_{n, i k}^{ \pm}+v_{i k}^{ \pm} \times F Y_{i}^{ \pm} \times R_{i j k}^{ \pm} \times D_{n, i k}^{ \pm}, \quad \forall i, j, k \\
& 0 \leq Z_{n, i j k}^{ \pm} \leq E_{n, i j k}^{ \pm}, \quad \forall i, j, k \\
& E_{n, i j k}^{ \pm}-Z_{n, i j k}^{ \pm} \leq S_{n, i k}^{ \pm}, \quad \forall i, j, k \\
& \sum_{i=1}^{I} S_{n, i k}^{ \pm} \leq T_{n, k}^{ \pm}, \quad \forall k \\
& E_{p, i j k}^{ \pm}=\mu_{i k}^{ \pm} \times L X_{i}^{ \pm} \times R_{i j k}^{ \pm} \times C_{p, i k}^{ \pm}+v_{i k}^{ \pm} \times F Y_{i}^{ \pm} \times R_{i j k}^{ \pm} \times D_{p, i k}^{ \pm}, \quad \forall i, j, k \\
& 0 \leq Z_{p, i j k}^{ \pm} \leq E_{p, i j k}^{ \pm}, \quad \forall i, j, k \\
& E_{p, i j k}^{ \pm}-Z_{p, i j k}^{ \pm} \leq S_{p, i k}^{ \pm}, \quad \forall i, j, k \\
& \sum_{i=1}^{I} S_{p, i k}^{ \pm} \leq T_{p, k}^{ \pm}, \quad \forall k \\
& \sum_{i=1}^{I}\left(L B_{i}^{ \pm} L X_{i}^{ \pm}+F B_{i}^{ \pm} F Y_{i}^{ \pm}\right)-\sum_{i=1}^{I} \sum_{j=1}^{J} \sum_{k=1}^{K} P_{i j k}\left(C N_{i k}^{ \pm} Z_{n, i j k}^{ \pm}+C P_{i k}^{ \pm} Z_{p, i j k}^{ \pm}\right) \geq \Omega_{q}^{ \pm}-U_{q} w_{i j k, q} \quad \forall i, j, k, q \\
& \sum_{i=1}^{I}\left(L B_{i}^{ \pm} L X_{i}^{ \pm}+F B_{i}^{ \pm} F Y_{i}^{ \pm}\right)-\sum_{i=1}^{I} \sum_{j=1}^{J} \sum_{k=1}^{K} P_{i j k}\left(C N_{i k}^{ \pm} Z_{n, i j k}^{ \pm}+C P_{i k}^{ \pm} Z_{p, i j k}^{ \pm}\right)<\Omega_{q}^{ \pm}+U_{q}\left(1-w_{i j k, q}\right) \quad \forall i, j, k, q \\
& L X_{i}^{-}+\Delta L X_{i} G_{i} \geq 0, \quad 0 \leq G_{i} \leq 1, \forall i \\
& F Y_{i}^{-}+\Delta F Y_{i} H_{i} \geq 0, \quad 0 \leq H_{i} \leq 1, \forall i .
\end{aligned}
$$


Next, interactive two-step algorithm proposed by Huang et al. [28] is used for converting model (25) into two deterministic submodels, which are corresponding to the upper and lower bounds of objective function value, respectively. Since objective function of model (25) is to maximize net system benefit, model (26) corresponding to $f^{+}$will be formulated firstly as follows:

$$
\begin{aligned}
& \text { Maximize } f^{+}=\sum_{i=1}^{I}\left(L B_{i}^{+}\left(L X_{i}^{-}+\Delta L X_{i} G_{i}\right)+F B_{i}^{+}\left(F Y_{i}^{-}+\Delta F Y_{i} H_{i}\right)\right)-\sum_{i=1}^{I} \sum_{j=1}^{J} \sum_{k=1}^{K} P_{i j k}\left(C N_{i k}^{-} Z_{n, i j k}^{-}+C P_{i k}^{-} Z_{p, i j k}^{-}\right)-\alpha_{m} \sum_{i=1}^{I} \sum_{j=1}^{J} \sum_{k=1}^{K} \sum_{q=1}^{Q} P_{i j k} w_{i j k, q} \\
& \text { Subject to: } \quad L X_{i}^{-}+\Delta L X_{i} G_{i}+F Y_{i}^{-}+\Delta F Y_{i} H_{i}=A_{i}, \quad \forall i \\
& E_{n, i j k}^{-}=\mu_{i k}^{-} \times\left(L X_{i}^{-}+\Delta L X_{i} G_{i}\right) \times R_{i j k}^{-} \times C_{n, i k}^{-}+v_{i k}^{-} \times\left(F Y_{i}^{-}+\Delta F Y_{i} H_{i}\right) \times R_{i j k}^{-} \times D_{n, i k}^{-}, \quad \forall i, j, k \\
& 0 \leq Z_{n, i j k}^{-} \leq E_{n, i j k}^{-}, \quad \forall i, j, k \\
& E_{n, i j k}^{-}-Z_{n, i j k}^{-} \leq S_{n, i k}^{-}, \quad \forall i, j, k \\
& \sum_{i=1}^{I} S_{n, i k}^{-} \leq T_{n, k}^{-}, \quad \forall k \\
& E_{p, i j k}^{-}=\mu_{i k}^{-} \times\left(L X_{i}^{-}+\Delta L X_{i} G_{i}\right) \times R_{i j k}^{-} \times C_{p, i k}^{-}+v_{i k}^{-} \times\left(F Y_{i}^{-}+\Delta F Y_{i} H_{i}\right) \times R_{i j k}^{-} \times D_{p, i k}^{-}, \quad \forall i, j, k \\
& 0 \leq Z_{p, i j k}^{-} \leq E_{p, i j k}^{-}, \quad \forall i, j, k \\
& E_{p, i j k}^{-}-Z_{p, i j k}^{-} \leq S_{p, i k}^{-}, \quad \forall i, j, k \\
& \sum_{i=1}^{I} S_{p, i k}^{-} \leq T_{p, k}^{-}, \quad \forall k \\
& \sum_{i=1}^{I}\left(L B_{i}^{+}\left(L X_{i}^{-}+\Delta L X_{i} G_{i}\right)+F B_{i}^{+}\left(F Y_{i}^{-}+\Delta F Y_{i} H_{i}\right)\right)-\sum_{i=1}^{I} \sum_{j=1}^{J} \sum_{k=1}^{K} P_{i j k}\left(C N_{i k}^{-} Z_{n, i j k}^{-}+C P_{i k}^{-} Z_{p, i j k}^{-}\right) \geq \Omega_{q}^{+}-U_{q} w_{i j k, q} \quad \forall i, j, k, q \\
& \sum_{i=1}^{I}\left(L B_{i}^{+}\left(L X_{i}^{-}+\Delta L X_{i} G_{i}\right)+F B_{i}^{+}\left(F Y_{i}^{-}+\Delta F Y_{i} H_{i}\right)\right)-\sum_{i=1}^{I} \sum_{j=1}^{J} \sum_{k=1}^{K} P_{i j k}\left(C N_{i k}^{-} Z_{n, i j k}^{-}+C P_{i k}^{-} Z_{p, i j k}^{-}\right)<\Omega_{q}^{+}+U_{q}\left(1-w_{i j k, q}\right) \quad \forall i, j, k, q \\
& L X_{i}^{-}+\Delta L X_{i} G_{i} \geq 0, \quad 0 \leq G_{i} \leq 1, \forall i \\
& F Y_{i}^{-}+\Delta F Y_{i} H_{i} \geq 0, \quad 0 \leq H_{i} \leq 1, \forall i .
\end{aligned}
$$

Decision variables, including $G_{i}, H_{i}, L X_{i, \mathrm{opt}}, F Y_{i, \mathrm{opt}}, Z_{n, i j k}^{-}$, and $Z_{p, i j k}^{-}$, are obtained through solving model (26) such that model (27) corresponding to $f^{-}$is formulated as follows:

$$
\begin{array}{ll}
\text { Maximize } \quad & f^{-}=\sum_{i=1}^{I}\left(L B_{i}^{-} L X_{i, \mathrm{opt}}+F B_{i}^{-} F Y_{i, \mathrm{opt}}\right)-\sum_{i=1}^{I} \sum_{j=1}^{J} \sum_{k=1}^{K} P_{i j k}\left(C N_{i k}^{+} Z_{n, i j k}^{+}+C P_{i k}^{+} Z_{p, i j k}^{+}\right)-\alpha_{m} \sum_{i=1}^{I} \sum_{j=1}^{J} \sum_{k=1}^{K} \sum_{q=1}^{Q} P_{i j k} w_{i j k, q} \\
\text { Subject to: } \quad & E_{n, i j k}^{+}=\mu_{i k}^{+} \times L X_{i, \mathrm{opt}} \times R_{i j k}^{+} \times C_{n, i k}^{+}+v_{i k}^{+} \times F Y_{i, \mathrm{opt}} \times R_{i j k}^{+} \times D_{n, i k}^{+}, \quad \forall i, j, k \\
& Z_{n, i j k}^{-} \leq Z_{n, i j k}^{+} \leq E_{n, i j k}^{+}, \quad \forall i, j, k \\
& E_{n, i j k}^{+}-Z_{n, i j k}^{+} \leq S_{n, i k}^{+}, \quad \forall i, j, k \\
& \sum_{i=1}^{I} S_{n, i k}^{+} \leq T_{n, k}^{+}, \quad \forall k \\
& E_{p, i j k}^{+}=\mu_{i k}^{+} \times L X_{i, \mathrm{opt}} \times R_{i j k}^{+} \times C_{p, i k}^{+}+v_{i k}^{+} \times F Y_{i, \mathrm{opt}} \times R_{i j k}^{+} \times D_{p, i k}^{+}, \quad \forall i, j, k \\
& Z_{p, i j k}^{-} \leq Z_{p, i j k}^{+} \leq E_{p, i j k}^{+}, \quad \forall i, j, k
\end{array}
$$




$$
\begin{aligned}
& E_{p, i j k}^{+}-Z_{p, i j k}^{+} \leq S_{p, i k}^{+}, \quad \forall i, j, k \\
& \sum_{i=1}^{I} S_{p, i k}^{+} \leq T_{p, k}^{+}, \quad \forall k \\
& \sum_{i=1}^{I}\left(L B_{i}^{-} L X_{i, \mathrm{opt}}+F B_{i}^{-} F Y_{i, \mathrm{opt}}\right)-\sum_{i=1}^{I} \sum_{j=1}^{J} \sum_{k=1}^{K} P_{i j k}\left(C N_{i k}^{+} Z_{n, i j k}^{+}+C P_{i k}^{+} Z_{p, i j k}^{+}\right) \geq \Omega_{q}^{-}-U_{q} w_{i j k, q} \quad \forall i, j, k, q \\
& \sum_{i=1}^{I}\left(L B_{i}^{-} L X_{i, \mathrm{opt}}+F B_{i}^{-} F Y_{i, \mathrm{opt}}\right)-\sum_{i=1}^{I} \sum_{j=1}^{J} \sum_{k=1}^{K} P_{i j k}\left(C N_{i k}^{+} Z_{n, i j k}^{+}+C P_{i k}^{+} Z_{p, i j k}^{+}\right)<\Omega_{q}^{-}+U_{q}\left(1-w_{i j k, q}\right) \quad \forall i, j, k, q .
\end{aligned}
$$

Finally, a variety of solutions of objective function value and decision variables under different weighted coefficients are obtained through solving above two models; that is, $f_{\text {opt }}^{ \pm}=\left[f_{\text {opt }}^{-}, f_{\text {opt }}^{+}\right], G_{i, \text { opt }}, H_{i, \text { opt }}, L X_{i, \text { opt }}, F Y_{i, \text { opt }}, Z_{n, i j k, \text { opt }}^{ \pm}=$ $\left[Z_{n, i j k, \mathrm{opt}}^{-}, Z_{n, i j k, \mathrm{opt}}^{+}\right], Z_{p, i j k, \mathrm{opt}}^{ \pm}=\left[Z_{p, i j k, \mathrm{opt}}^{-}, Z_{p, i j k, \mathrm{opt}}^{+}\right], S_{n, i k}^{ \pm}=$ $\left[S_{n, i k, \mathrm{opt}}^{-}, S_{n, i k, \mathrm{opt}}^{+}\right]$, and $S_{p, i k}^{ \pm}=\left[S_{p, i k, \mathrm{opt}}^{-}, S_{p, i k, \mathrm{opt}}^{+}\right]$, respectively.

Figure 2 shows general framework of RBITSP model, where optimization software LINGO 12.0 is used to solve model due to its user-friendly interface, easy-to-edit language, and quick computational speed. The computational time of solving model is within a few seconds. Detailed procedures of formulating and solving RBITSP model are summarized as follows.

Step 1. Identify uncertain variables and acquire related probabilistic distribution and discrete-interval information.

Step 2. Formulate an ITSP model.

Step 3. Incorporate measure of financial risk into ITSP model, including target level of profit $\Omega_{q}^{ \pm}$and binary variable $w_{i j k, q}$, such that RBITSP model is obtained.

Step 4. Transform RBITSP model into ITSP model through introducing variable $U_{q}$, which is predefined upper bound of profit target.

Step 5. Reformulate ITSP model by introducing $L X_{i}^{ \pm}=$ $L X_{i}^{-}+\Delta L X_{i} G_{i}$, where $\Delta L X_{i}=L X_{i}^{+}-L X_{i}^{-}$and $G_{i} \in[0,1]$; $F Y_{i}^{ \pm}=F Y_{i}^{-}+\Delta F Y_{i} H_{i}$, where $\Delta F Y_{i}=F Y_{i}^{+}-F Y_{i}^{-}$and $H_{i} \in[0,1]$.

Step 6. Transform original ITSRP model into two submodels, which correspond to $f^{+}$and $f^{-}$, respectively.

Step 7. Solve two submodels and generate final solutions of $f_{\mathrm{opt}}^{ \pm}=\left[f_{\mathrm{opt}}^{-}, f_{\mathrm{opt}}^{+}\right], G_{i, \mathrm{opt}}, H_{i, \mathrm{opt}}, L X_{i, \mathrm{opt}}, F Y_{i, \mathrm{opt}}, Z_{n, i j k, \mathrm{opt}}^{ \pm}=$ $\left[Z_{n, i j k, \mathrm{opt}}^{-}, Z_{n, i j k, \mathrm{opt}}^{+}\right], Z_{p, i j k, \mathrm{opt}}^{ \pm}=\left[Z_{p, i j k, \mathrm{opt}}^{-}, Z_{p, i j k, \mathrm{opt}}^{+}\right], S_{n, i k}^{ \pm}=$ $\left[S_{n, i k, \mathrm{opt}}^{-}, S_{n, i k, \mathrm{opt}}^{+}\right]$, and $S_{p, i k}^{ \pm}=\left[S_{p, i k, \mathrm{opt}}^{-}, S_{p, i k, \mathrm{opt}}^{+}\right]$, respectively.

\section{Result Analysis and Discussion}

4.1. Result Analysis. Table 4 reflects a part of solutions generated by proposed RBITSP model at fixed alpha value (i.e., weight coefficient) and various omega values (i.e., predetermined profit target), which include objective function values and decision variables. Herein, alpha value reflects the decision maker's attitudes to financial risk and is an acceptable extent for system revenue not reaching predesigned target value under various situations, where the increase in alpha values means financial risk is emphasized. In this study, we have tested different sets of alpha values and found that varying trends of objective function values and most of nonzero decision variables under different sets of alpha values are similar. Thus, we consider alpha values of 1 and 1000 as representative and use them for further result analysis. As for omega values, they are designed and determined based on calculated system incomes under various situations. Finally, four situations are selected (i.e., low, low-medium, medium, and high income) and each situation includes four target values which are expressed as interval forms, where four target values under the low condition are $[8,13],[10,15]$, $[12,17]$, and $[14,19] \times 10^{6} \$$, respectively; those under the low-medium condition are $[10,17],[12,19],[14,21]$, and $[16,23] \times 10^{6} \$$, respectively; those under the medium condition are $[12,19],[14,21],[16,23]$, and $[18,25] \times 10^{6} \$$, respectively; those under the high condition are $[14,21]$, $[16,23],[18,25]$, and $[20,27] \times 10^{6} \$$, respectively. Since RBITSP was developed through incorporating financial risk measure into ITSP model, the solutions show characteristics of both methods.

4.1.1. The Trend Analysis in Decision Variables. Under the influences imposed by ILP solution algorithm, decision variables and objective function are expressed as interval numbers. For example, when omega values are under the low condition at a fixed alpha of 1 , variation range of expected incomes is $[38.80,52.68] \times 10^{6} \$$. Among them, solutions (i.e., expected income values and planting areas) corresponding to the lower bounds were obtained under the conditions when parameters such as planting return, available land area, and allowed pollutant discharge amounts 


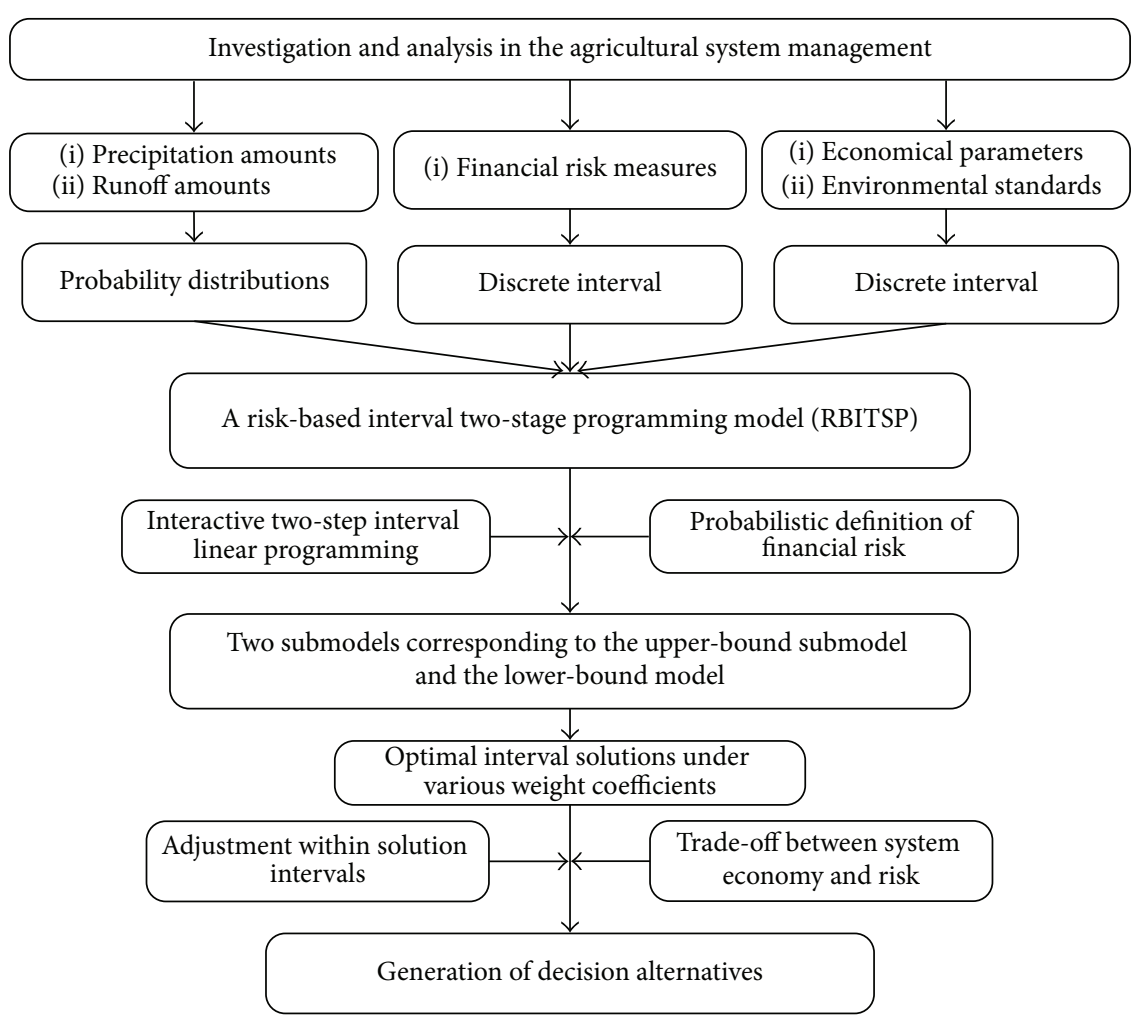

FIGURE 2: Formulation and solution framework of RBITSP model.

TABLE 4: Part of solutions from RBITSP model under the coefficient of alpha $=1$.

\begin{tabular}{|c|c|c|c|c|c|}
\hline \multirow{2}{*}{ Decision variables } & \multicolumn{5}{|c|}{ Agricultural zone $(i)$} \\
\hline & $i=1$ & $i=2$ & $i=3$ & $i=4$ & $i=5$ \\
\hline \multicolumn{6}{|c|}{ The target values (i.e., omega values) are under the low condition } \\
\hline The amounts of the $\mathrm{TN}(\mathrm{kg})$ & {$[245602,341569]$} & {$[89858,171540]$} & {$[287864,437783]$} & {$[121050,169887]$} & {$[197255,266149]$} \\
\hline The amounts of the TP $(\mathrm{kg})$ & {$[25022,34333]$} & {$[18181,26359]$} & {$[26922,39707]$} & {$[12008,16959]$} & {$[19867,27696]$} \\
\hline The cropland area (ha) & 4240 & 3292.78 & 3876.86 & 2500 & 4160 \\
\hline The forestland area (ha) & 3080 & 7167.22 & 8613.15 & 2600 & 2150 \\
\hline \multicolumn{6}{|c|}{ The target values (i.e., omega values) are under the low-medium condition } \\
\hline The amounts of the TN $(\mathrm{kg})$ & {$[241417,341113]$} & {$[76574,153242]$} & {$[285988,431687]$} & {$[121050,169631]$} & {$[191256,266149]$} \\
\hline The amounts of the TP $(\mathrm{kg})$ & {$[25023,34310]$} & {$[17306,24749]$} & {$[25467,38197]$} & {$[11986,16959]$} & {$[19867,27696]$} \\
\hline The cropland area (ha) & 4240 & 2800 & 3845.86 & 2500 & 4160 \\
\hline The cropland area (ha) & 3080 & 7600 & 8644.14 & 2600 & 2150 \\
\hline \multicolumn{6}{|c|}{ The target values (i.e., omega values) are under the medium condition } \\
\hline The amounts of the TN $(\mathrm{kg})$ & {$[241417,341113]$} & {$[38962,136301]$} & {$[323600,448628]$} & {$[121050,169631]$} & {$[191256,266149]$} \\
\hline The amounts of the TP $(\mathrm{kg})$ & {$[25022,34310]$} & {$[13496,21563]$} & {$[29277,41383]$} & {$[11986,16959]$} & {$[19867,27696]$} \\
\hline The cropland area (ha) & 4240 & 2800 & 3845.86 & 2500 & 4160 \\
\hline The cropland area (ha) & 3080 & 7600 & 8644.14 & 2600 & 2150 \\
\hline \multicolumn{6}{|c|}{ The target values (i.e., omega values) are under the high condition } \\
\hline The amounts of the TN $(\mathrm{kg})$ & {$[236961,336658]$} & {$[38963,136301]$} & {$[326268,452678]$} & {$[121050,169627]$} & {$[191256,266149]$} \\
\hline The amounts of the TP $(\mathrm{kg})$ & {$[25021,34310]$} & {$[12067,20134]$} & {$[30672,42766]$} & {$[11987,16959]$} & {$[19867,27696]$} \\
\hline The cropland area (ha) & 4240 & 2800 & 3840 & 2500 & 4160 \\
\hline The cropland area (ha) & 3080 & 7600 & 8650 & 2600 & 2150 \\
\hline
\end{tabular}




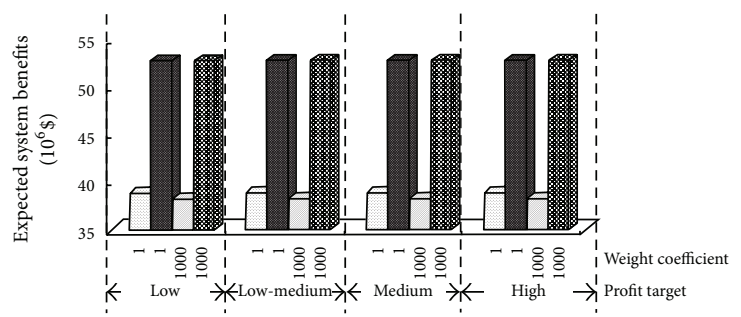

- The lower-bound objective function values under alpha $=1$

- The upper-bound objective function values under alpha $=1$

- The lower-bound objective function values under alpha $=1000$

- The upper-bound objective function values under alpha $=1000$

FIGURE 3: Expected objective values of RBITSP model.

reached their lower bounds, and other parameters such as economical punishment on excessive pollutant emissions, surface runoff coefficient, and pollutant emission coefficient reached to their upper bounds. It is concluded that decision alternatives corresponding to the lower bounds show poor performance on economical aspect, which is more in favor of resource conservation and water-quality protection. On the contrary, decision schemes of upper bounds are beneficial in system profits, which are accompanied with resource shortage and water pollution. Interval forms of objective functions and decision variables can effectively reflect tradeoff between economic revenues and protection of resources and environment, which could facilitate decision makers to generate agricultural production patterns based on their judgment and preference.

Unlike ITSP model, in this study, the measures of financial risk were incorporated into objective function, and different omega and alpha values were designed for reflecting trade-off between system economy and risk. As shown in Table 4, the variation of these two parameters has great impacts on obtained solutions. Firstly, with the increase of omega value (when alpha value is fixed), cropland area in subzones 2 and 3 would decrease and forestland area would increase. For example, when alpha takes the value of 1 , cropland areas of subzone 2 are 3292.78, 2800, 2800, and 2800 ha while forestland areas are 7167.22, 7600, 7600, and 7600 ha, respectively. Similarly, farmland areas of subzone 3 are $3876.86,3845.86,3845.86$, and 3840 ha while its forestland areas are $8613.15,8644.14,8644.14$, and 8650 ha, respectively. It is mainly because that increase of omega will result in increase in predesigned target values of system profits. Although the revenue of farmland is higher than that of forestland, nevertheless, runoff volume and pollutant discharge amounts of farmland are also much higher. This will lead to the fact that punishments of farmland deriving from excessive pollutant discharge are higher than those of forestland. In order to avoid penalty cost, farm areas would decrease while forestland areas become increased.

4.1.2. The Trend Analysis in Objective Function. As shown in Figure 3, parameters above-mentioned have great impacts not only on decision variables, but also on expected value of system profits. Under the influences of two indicators, expected profits exhibit certain variation tendency. Firstly, when alpha value is fixed, with the increase of omega, expected system profits would increase. For example, at a weighted coefficient of alpha $=1$, expected revenue is [38.80, 52.68], [39.00, 52.71], [39.01, 52.73], and [39.02, 52.74] $\times 10^{6} \$$. Under a value of alpha $=1000$, expected income is [38.27, 52.55], [38.45, 52.57], [38.45, 52.57], and [38.45, 52.57] $\times$ $10^{6} \$$. Similarly, when omega value is fixed, expected profit would decrease with decrease of alpha value. The profits at two alpha values under the low condition are [38.80, $52.68]$ and $[38.27,52.55] \times 10^{6} \$$, respectively; those under the high situation are $[39.02,52.73]$ and $[38.45,52.57] \times 10^{6} \$$, respectively. This is because that the increase of omega value requires expected system profits under different circumstance increase as much as possible in order to avoid risk, while alpha values remain unchanged; thus, total expected incomes would increase. As for the increase of alpha value, it will emphasize importance on financial risks under different situations, which cannot guarantee the maximization of total expected incomes, leading to the decrease of total expected revenues.

The variations above-mentioned effectively reflect tradeoff between system profits and risks. The increase in expected profits can only guarantee average profit level of management system. Nevertheless, it cannot ensure that system profits under some specific conditions also meet requirement simultaneously. In contrast, economic benefit may be loss in order to make system profits under specific conditions reach predetermined standard. Under the context of global warming, frequent occurrences of extreme climate event and flood disasters associated with Tai Lake watershed may exacerbate failure risk in decision-making process. RBITSP model is capable of reflecting risk measure and generating a variety of solutions realizing balance of system-profit targets and system-failure risk. Decision alternatives at the low alpha and high omega values are suitable in avoiding risk and providing instructions for local authorities. Generally, RBITSP model formulated in this study has realized improvement and its feasibility and practicability are also obviously enhanced. The introduction of two indicators omega and alpha, on the one hand, can overcome disadvantage that ITSP model only emphasizes the maximization of expected profits and neglects unsatisfied values under specific situations. On the other hand, the adjustment of parameters effectively reflects trade-off between system profits and risks, which can not only enrich the diversity of generated solution but also provide basis for decision makers. This model is suitable in tackling agricultural production management issue of subareas in Tai Lake watershed. It is extremely useful for local managers under circumstance of global warming and frequent occurrence of extreme weather events.

4.2. Discussion. In order to better reflect the advantages of proposed RBITSP model, in this study, ITSP model was formulated for tackling the same problem. Here, constraints related to financial risk and punishment caused by profit lower than predefined target in objective functions were deleted. The objective function obtained from ITSP model 
is $[39.02,52.73] \times 10^{6} \$$, which is included within interval solutions generated by RBITSP model, which indicates that solution from ITSP model is special case. However, RBITSP model also has some drawbacks in theoretical framework and practical applications which need further improvements. Firstly, the determination of omega values has great impacts on obtained model solutions. In this study, omega value was determined by calculating system profits under various conditions, which represent different backgrounds. How to design rational omega values is critical for generating accurate decision schemes. Secondly, the introductions of two indicators (omega and alpha) also bring out a question, which is how to select reasonable solutions. Since every solution has its own specific meanings, selection of evaluation criteria and methods will be critical for determining management alternatives. Finally, other uncertain optimization methods, such as FMP, could be incorporated into model for handling more-complex issue.

\section{Conclusions}

In this study, a risk-based interval two-stage programming model was developed for designing and generating an economy-effective, environmentally friendly, and risk-averse agricultural production pattern. Compared with general ITSP model, main advantage of proposed model was that it provided enough attention on financial risk under various probabilistic levels, rather than only being concentrated on economic benefit. The way was suitable in generating more stable solutions, which realized balance between system economy and reliability. Applied results of RBITSP model in agricultural management system with Tai Lake watershed showed that it could be a base for designing and determining agricultural development schemes. Decision alternative (its expected profit reached $[38.45,52.57] \times 10^{6} \$$ ) under the high alpha and omega values was recommended to be a decision base due to its balanced characteristic. In the future, some improvements, including selection of critical indicators, the incorporation of multicriteria evaluation technology, and other uncertain optimization approaches, deserved to be further studied.

\section{Competing Interests}

The authors declare that there are no competing interests regarding the publication of this paper.

\section{Acknowledgments}

This research was supported by the Fundamental Research Funds for the Central Universities (Grant no. 2015ZZD08).

\section{References}

[1] Y. Xu and X. S. Qin, "Agricultural effluent control under uncertainty: an inexact double-sided fuzzy chance-constrained model," Advances in Water Resources, vol. 33, no. 9, pp. 997-1014, 2010.
[2] S. Karmakar and P. P. Mujumdar, "Grey fuzzy optimization model for water quality management of a river system," Advances in Water Resources, vol. 29, no. 7, pp. 1088-1105, 2006.

[3] J. H. Ellis, "Stochastic water quality optimization using imbedded chance constraints," Water Resources Research, vol. 23, no. 12, pp. 2227-2238, 1987.

[4] N.-B. Chang, C. G. Wen, and S. L. Wu, "Optimal management of environmental and land resources in a reservoir watershed by multiobjective programming," Journal of Environmental Management, vol. 44, no. 2, pp. 145-161, 1995.

[5] R. Kerachian and M. Karamouz, "A stochastic conflict resolution model for water quality management in reservoir-river systems," Advances in Water Resources, vol. 30, no. 4, pp. 866882, 2007.

[6] B. Julien, "Water quality management with imprecise information," European Journal of Operational Research, vol. 76, no. 1, pp. 15-27, 1994.

[7] N.-B. Chang, H. W. Chen, and S. K. Ning, "Identification of river water quality using the fuzzy synthetic evaluation approach," Journal of Environmental Management, vol. 63, no. 3, pp. 293$305,2001$.

[8] W. Zhang and X. J. Wang, "Modeling for point-nonpoint source effluent-trading: perspective of nonpoint sources regulation in Canada," Science of the Total Environment, vol. 292, no. 3, pp. 167-176, 2002.

[9] S. Ghosh and P. P. Mujumdar, "Risk minimization in water quality control problems of a river system," Advances in Water Resources, vol. 29, no. 3, pp. 458-470, 2006.

[10] L. J. Wang, W. Meng, H. C. Guo, Z. X. Zhang, Y. Liu, and Y. Y. Fan, "An interval fuzzy multiobjective watershed management model for the Lake Qionghai watershed, China," Water Resources Management, vol. 20, no. 5, pp. 701-721, 2006.

[11] S. H. Ghodsi, R. Kerachian, S. M. Estalaki, M. R. Nikoo, and Z. Zahmatkesh, "Developing a stochastic conflict resolution model for urban runoff quality management: application of info-gap and bargaining theories," Journal of Hydrology, vol. 533, pp. 200-212, 2016.

[12] S. Rehana and P. P. Mujumdar, "An imprecise fuzzy risk approach for water quality management of a river system," Journal of Environmental Management, vol. 90, no. 11, pp. 36533664, 2009.

[13] M. O. Ribaudo and C. J. Nickerson, "Agriculture and water quality trading: exploring the possibilities," Journal of Soil and Water Conservation, vol. 64, no. 1, pp. 1-7, 2009.

[14] W. Li, M. Liu, S. Z. Wu, and Y. Xu, "An inexact optimization model associated with two robust programming approaches for water resources management," International Journal of Environmental Science and Technology, vol. 12, no. 7, pp. 24012414, 2015.

[15] A. Tavakoli, M. R. Nikoo, R. Kerachian, and M. Soltani, "River water quality management considering agricultural return flows: application of a nonlinear two-stage stochastic fuzzy programming," Environmental Monitoring and Assessment, vol. 187, no. 4, 2015.

[16] M. Li, P. Guo, and C. F. Ren, "Water resources management models based on two-level linear fractional programming method under uncertainty," Journal of Water Resources Planning and Management, vol. 141, no. 9, Article ID 05015001, 2015.

[17] X. Zhu, D. Li, and L. F. Rodriguez, "An agent-based simulation model of a nutrient trading market for natural resources management," Mathematical and Computer Modelling, vol. 54, no. 3-4, pp. 987-994, 2011. 
[18] G. H. Huang and D. P. Loucks, "An inexact two-stage stochastic programming model for water resources management under uncertainty," Civil Engineering and Environmental Systems, vol. 17, no. 2, pp. 95-118, 2000.

[19] C. Y. Li and L. Zhang, "An inexact two-stage allocation model for water resources management under uncertainty," Water Resources Management, vol. 29, no. 6, pp. 1823-1841, 2015.

[20] N. Zhang, Y. P. Li, W. W. Huang, and J. Liu, "An inexact twostage water quality management model for supporting sustainable development in a rural system," Journal of Environmental Informatics, vol. 24, no. 1, pp. 52-64, 2014.

[21] Y. Huang, Y. P. Li, X. Chen, and Y. G. Ma, "Optimization of the irrigation water resources for agricultural sustainability in Tarim River Basin, China," Agricultural Water Management, vol. 107, pp. 74-85, 2012.

[22] M. Liu, G. H. Huang, R. F. Liao, Y. P. Li, and Y. L. Xie, "Fuzzy two-stage non-point source pollution management model for agricultural systems-a case study for the Lake Tai Basin, China," Agricultural Water Management, vol. 121, pp. 27-41, 2013.

[23] M. Li and P. Guo, "A coupled random fuzzy two-stage programming model for crop area optimization-a case study of the middle Heihe River basin, China," Agricultural Water Management, vol. 155, pp. 53-66, 2015.

[24] S. Y. Xia, Study on comprehensive management in Taihu Lake Basin water pollution [M.S. thesis], Soochow University, 2012 (Chinese).

[25] L. X. Shi, X. Y. Li, N. Wang, and L. Z. Yang, "Technologies of NPS pollution control in Taihu Basin and countermeasures," China Water Resources, pp. 11-14, 2003 (Chinese).

[26] K. Cao and X. Y. Ye, "Coarse-grained parallel genetic algorithm applied to a vector based land use allocation optimization problem: the case study of Tongzhou Newtown, Beijing, China," Stochastic Environmental Research and Risk Assessment, vol. 27, no. 5, pp. 1133-1142, 2013.

[27] A. Barbaro and M. J. Bagajewicz, "Managing financial risk in planning under uncertainty," Process Systems Engineering, vol. 50, pp. 963-989, 2004.

[28] G. H. Huang, B. W. Baetz, and G. G. Patry, "A grey linear programming approach for municipal solid waste management planning under uncertainty," Civil Engineering Systems, vol. 9, no. 4, pp. 319-335, 1992. 


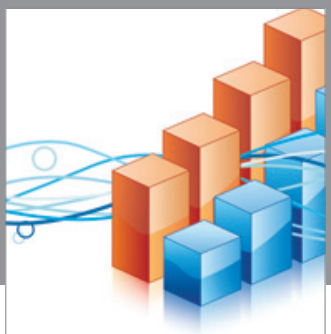

Advances in

Operations Research

vatem alat4

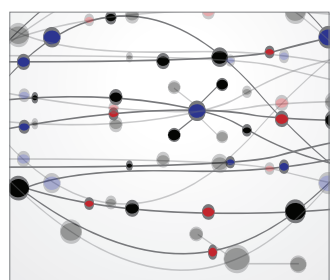

\section{The Scientific} World Journal
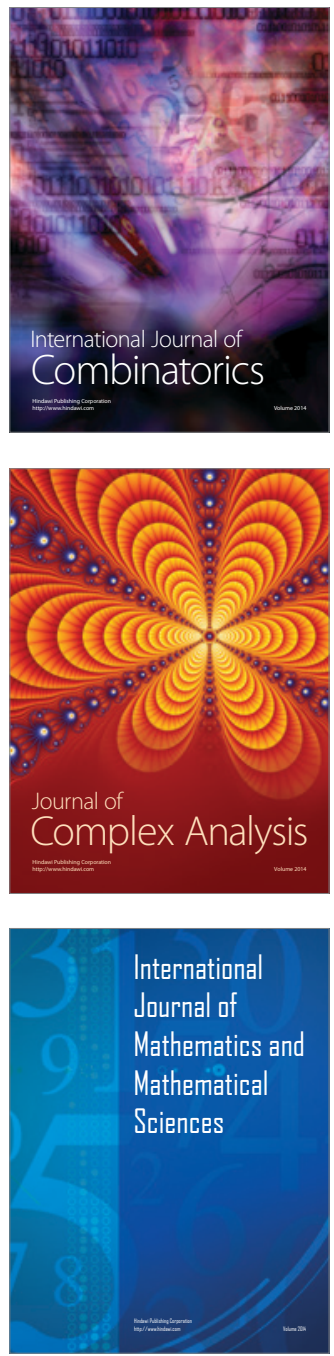
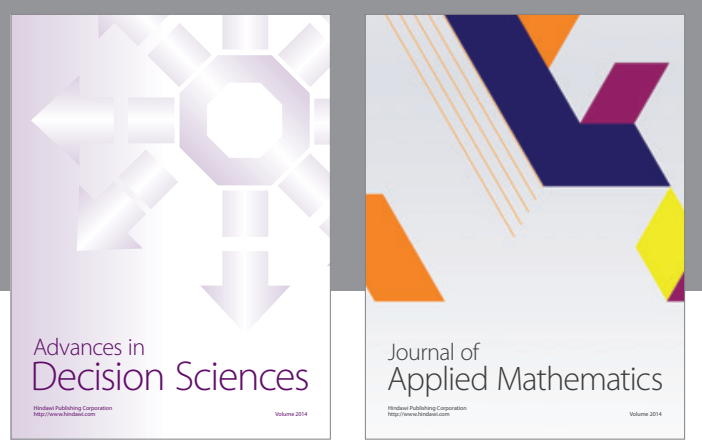

Algebra

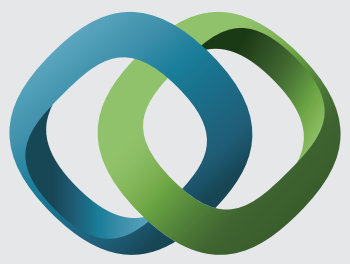

\section{Hindawi}

Submit your manuscripts at

http://www.hindawi.com
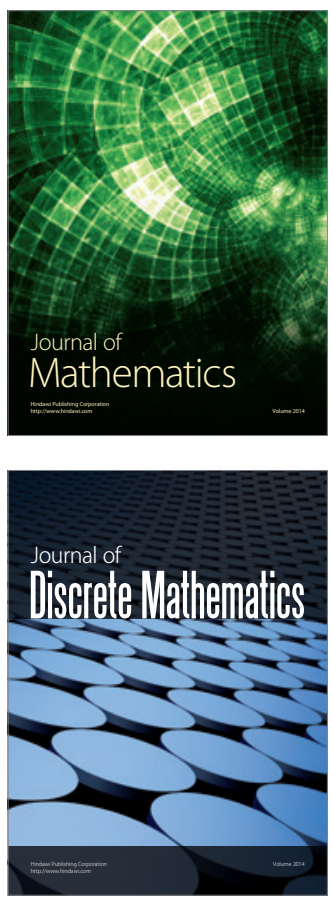

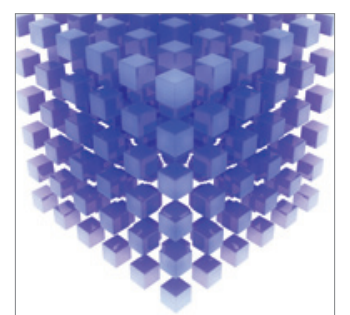

Mathematical Problems in Engineering
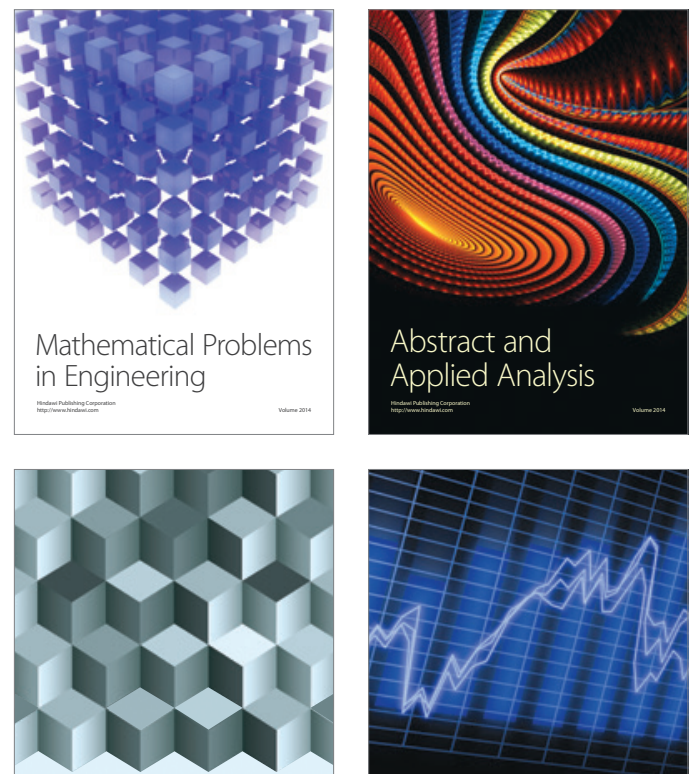

Journal of

Function Spaces

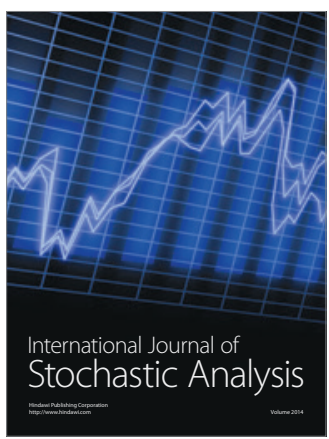

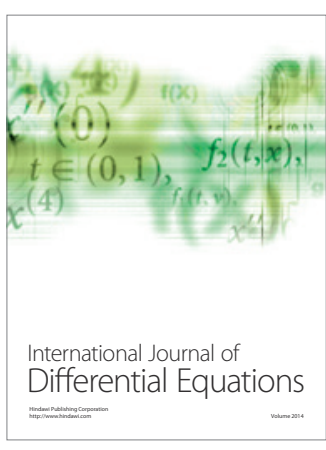
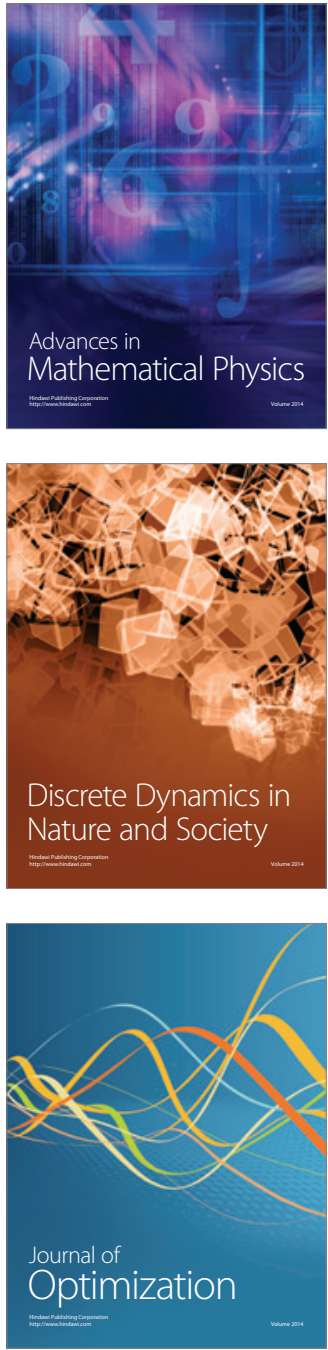